Check for updates

Cite this: RSC Adv., 2018, 8, 6581

Received 1st December 2017

Accepted 26th January 2018

DOI: $10.1039 / c 7 r a 12969 f$

rsc.li/rsc-advances

\section{Fabrication of a hyaluronic acid conjugated metal organic framework for targeted drug delivery and magnetic resonance imaging}

\author{
Fangpeng Shu, ${ }^{a}$ Daojun Lv, ${ }^{a}$ Xian-Lu Song, ${ }^{b}$ Bin Huang, ${ }^{a}$ Chong Wang, ${ }^{a}$ Yuzhong $\mathrm{Yu}^{\mathrm{a}}$ \\ and Shan-Chao Zhao (iD *a
}

Since metal organic frameworks (MOF) have exhibited fascinating potential in biomedical applications, it is worthwhile to construct a MOF-based multifunctional drug delivery system. In the present study, the anticancer drug doxorubicin (DOX) was loaded into zeolitic imidazolate framework-8 (ZIF-8) via a onepot process. The formed DOX(AZIF- 8 was then coated with polydopamine, successively chelated with $\mathrm{Fe}^{3+}$ and conjugated with hyaluronic acid $(\mathrm{HA})$, finally resulting in a multifunctional $\mathrm{ZIF}-8$ nanocarrier. The characterization results confirmed the successful formation of the hybrid nanocarrier. $\mathrm{pH}$ responsive drug release of DOX was observed due to the innate $\mathrm{pH}$-dependent stability of ZIF-8. Importantly, the flow cytometry and confocal laser scanning microscope results both verified the targeting ability of DOX(aZIF-HA toward prostate cancer PC-3 cells. The improved therapeutic efficacy of DOX@ZIF-HA when compared to the inhibited group was also demonstrated. Furthermore, the chelation of $\mathrm{Fe}^{3+}$ by PDA makes the prepared DOX(aZIF-HA a good contrast agent for magnetic resonance (MR) imaging. Hence, we hope the constructed ZIF- 8 based multifunctional nanocarrier could be a candidate for cancer theranostics.

\section{Introduction}

As one of the most frequently used approaches for combating cancer, chemotherapy has always suffered some unbearable drawbacks, including non-specific toxicity toward normal cells, premature degradation in circulation and insufficient therapeutic efficacy. ${ }^{1}$ To address those problems, various nanocarriers, such as liposomes, ${ }^{2}$ polymer micelles, ${ }^{3}$ graphene ${ }^{4}$ and mesoporous silica nanoparticles, ${ }^{5}$ have been widely developed for packaging and delivering chemotherapeutic drugs. Nanocarriers can not only protect the drug from rapid clearance, but can also improve its accumulation at the tumor site through the enhanced permeability and retention (EPR) effect. ${ }^{6}$ However, currently explored nanocarriers still have a few shortcomings to conquer. For instance, the stability of organic nanocarriers is poor and they can only offer single functionality, ${ }^{7}$ while debates about the biocompatibility and biodegradability of inorganic nanocarriers have never stopped. ${ }^{8}$ The construction of organic/ inorganic hybrid nanocarriers could adequately integrate the functionality of inorganic nanomaterials with the biocompatibility of organic nanomaterials. ${ }^{9}$ Unfortunately, simple hybridization still fails to change their innate physiochemical

\footnotetext{
${ }^{a}$ Department of Urology, Nanfang Hospital, Southern Medical University, Guangzhou 510515, China. E-mail: zhaoshanchaosmu@sina.cn

${ }^{b}$ Department of Radiation Oncology, Affiliated Cancer Hospital \& Institute of Guangzhou Medical University, Guangzhou 510095, China
}

properties. As a consequence, the development of novel nanocarriers with a natural organic/inorganic constitution may strongly promote the translation process of nanomedicine.

Metal organic frameworks (MOFs) are highly porous materials composed of metal ions and organic linkers. ${ }^{10}$ In particular, nano-scale MOFs have drawn extensive attention owing to their high surface area, tunable shapes and pore sizes, and controllable surface functionalities. ${ }^{11}$ Built from zinc ions and imidazole units, zeolitic imidazolate framework-8 (ZIF-8) is one of the most widely investigated subclasses of MOFs for biomedical applications. ${ }^{12}$ It is well known that ZIF-8 can decompose under lower $\mathrm{pH}$ conditions, ${ }^{13}$ thus making it a good candidate for a $\mathrm{pH}$-responsive nanocarrier for drug delivery. For instance, Wang's group ${ }^{14}$ developed a facile two-step method to fabricate green fluorescent carbon nanodots@ZIF-8 with adjustable size and fluorescence intensity, then the prepared hybrid ZIF-8 was loaded with 5-fluorouracil and a pH-responsive drug release behavior was demonstrated. In another study, $\mathrm{He}$ et al. ${ }^{15}$ prepared CuS-encapsulated ZIF-8 and then the anticancer drug doxorubicin (DOX) was loaded into the hybrid ZIF-8 nanoparticles for combined therapy. However, the drug loading process in those studies was mostly accomplished in two steps: the formation of ZIF-8 and the absorption of the drug. This approach always suffers from low loading efficacy and poorly controlled release of the molecules. Very recently, Zou and coworkers ${ }^{16}$ proposed a one-pot synthetic procedure for the preparation of targeted molecule-encapsulated ZIF-8 in the 
aqueous phase. The results demonstrated that the assynthesized drug-loaded nanocrystal could also act as an efficient $\mathrm{pH}$-responsive drug delivery vehicle for chemotherapy. So this simple, scalable, and eco-friendly technique is likely to be preferable for the fabrication of drug-loaded nanocarriers when compared to conventional methods. ${ }^{17}$

On the other hand, the unsatisfactory therapeutic efficacy has always encouraged researchers to modify target molecules onto nanocarriers for improved efficacy. ${ }^{18}$ With regard to MOFs, various target molecules were also applied to improve their specific ability towards cancer cells. As an example, Sahu et al. ${ }^{19}$ used ZIF-8 to coat upconverting nanoparticles for cancer theranostics, onto which folic acid was also attached for targeted drug delivery. In that system, the internal upconverting nanoparticles acted as an imaging component, the outer layer ZIF-8 was loaded with 5-fluorouracil for chemotherapy and folic acid acted as a target molecule for enhancing the chemotherapeutic efficacy against HeLa cells. Besides, the fabrication of a theranostic nanoagent based on ZIF-8 is also favorable for precision nanomedicine. Recently, Wang et al. ${ }^{20}$ used ZIF-8 to encapsulate $\mathrm{Fe}_{3} \mathrm{O}_{4}$ nanoparticles and gold nanoclusters and then the anticancer drug doxorubicin was loaded into the cavity of ZIF-8 for chemotherapy. The obtained hybrid nanoplatform could not only deliver DOX into cancer cells, but also could be applied for magnetic resonance (MR) imaging and fluorescence imaging, thereby achieving simultaneous therapeutic and diagnostic functions. Considering the relatively complicated process of the above design, it is highly desirable to develop a facile strategy to construct a multifunctional theranostic nanoplatform.

Herein, we present a facile synthesis of a multifunctional nanocarrier based on ZIF-8 for cancer theranostics. Conveniently, DOX was encapsulated into the ZIF-8 structure (DOX@ZIF-8) via a one-pot synthesis process. Subsequently, polydopamine (PDA) was applied to modify DOX@ZIF-8 to provide it with an active surface via mussel-inspired polymerization. Furthermore, hyaluronic acid (HA), a widely reported targeting molecule, ${ }^{21}$ was conjugated onto DOX@ZIF-8 via ironmediated coordination. Even though a vast amount of nanocarriers have been functionalized with HA for targeted drug delivery, ${ }^{22,23}$ the modification of HA on ZIF-8 for targeted chemotherapy has not yet been reported to the best of our knowledge. So we think three considerations can be highlighted for this work: first, the one-pot drug loading process is more convenient than most reported MOF-based nanocarriers as described above; second, the facile modification of HA can facilitate its active targeted delivery into specific cancer cells; third and more importantly, the coordination interaction between $\mathrm{Fe}^{3+}$ and PDA demonstrates that it could be used as a $T_{1}$ contrast agent for MR imaging, making the prepared hybrid MOF-based nanocarrier a good candidate for theranostic nanoplatforms. The obtained DOX@ZIF-HA was systematically characterized. The DOX release behavior was investigated and the targeting ability of DOX@ZIF-HA toward prostate cancer cell line PC-3 cells was emphasized. Last, the in vitro MR imaging capability of DOX@ZIF-HA was also inspected. Thus, the constructed multifunctional nanocarrier possessed both therapeutic and diagnostic functions.

\section{Experimental section}

\subsection{Materials}

$\mathrm{Zn}\left(\mathrm{NO}_{2}\right)_{3} \cdot 6 \mathrm{H}_{2} \mathrm{O}$, 2-methylimidazole, dopamine hydrochloride and $\mathrm{FeCl}_{3} \cdot 6 \mathrm{H}_{2} \mathrm{O}$ were purchased from Sigma-Aldrich Trading Co., Ltd. Amino-poly(ethylene glycol) $\left(\right.$ PEG-NH $\left._{2}, M_{\mathrm{w}} \sim 2000 \mathrm{Da}\right)$ was received from JenKem Technology Co, Ltd. Doxorubicin hydrochloride was bought from Beijing Huafeng United Technology Co., Ltd. Roswell Park Memorial Institute (RPMI) 1640 medium, trypsin, fetal bovine serum (FBS) and penicillinstreptomycin solution were supplied by Hyclone (Thermo Scientific, USA). A cell counting kit-8 (CCK-8) was provided by Sigma-Aldrich Trading Co., Ltd. 4',6-Diamidino-2-phenylindole (DAPI) was received from Vector laboratories (Burlingame, CA). All other reagents were of analytical grade and used without further purification. Deionized (DI) water was used throughout all experiments.

\subsection{Synthesis of DOX-encapsulated ZIF-8}

Chemotherapeutic DOX was incorporated into the framework of ZIF-8 via a "one-pot" method. ${ }^{\mathbf{1 6}}$ Generally, DOX was dissolved in DI water at $4 \mathrm{mg} \mathrm{mL}{ }^{-1}$. Then $0.2 \mathrm{~g}(0.66 \mathrm{mmol})$ of $\mathrm{Zn}\left(\mathrm{NO}_{3}\right)_{2}{ }^{-}$ $\cdot 6 \mathrm{H}_{2} \mathrm{O}$ was dissolved in $0.8 \mathrm{~mL}$ water, into which $4 \mathrm{~mL}$ of DOX solution was added and vigorously stirred for $1 \mathrm{~min}$. After that $2 \mathrm{~g}$ 2-methylimidazole was dissolved in $8 \mathrm{~mL}$ water and this was slowly dropped into the mixture. The reaction was maintained at room temperature for $15 \mathrm{~min}$. The DOX@ZIF-8 nanoparticles were collected using high-speed centrifugation and washed with DI water three times.

\subsection{Preparation of polydopamine-coated DOX@ZIF-8}

To modify their inert surface, polydopamine (PDA) was coated onto DOX@ZIF-8 via mussel-inspired polymerization. ${ }^{24}$ Briefly, 20 mg DOX@ZIF-8 was ultrasonically dispersed in $20 \mathrm{~mL}$ Tris$\mathrm{HCl}$ solution ( $\mathrm{pH} 8.5,10 \mathrm{mM}$ ). Then $10 \mathrm{mg}$ dopamine hydrochloride was added and the reaction was continued for $4 \mathrm{~h}$. PDA-coated DOX@ZIF-8 was separated by centrifugation and washed with DI water.

\subsection{Modification of hyaluronic acid (HA)}

The target molecule HA was conjugated onto DOX@ZIF-8 via an $\mathrm{Fe}^{3+}$-mediated coordination reaction. ${ }^{25}$ Typically, $10 \mathrm{mg}$ DOX@ZIF-PDA was dispersed in $10 \mathrm{~mL}$ Tris-HCl solution (pH $8.5,10 \mathrm{mM}$ ). Then $0.1 \mathrm{~mL}$ of $\mathrm{FeCl}_{3}$ solution was added and stirred for $1 \mathrm{~h}$. Subsequently, $10 \mathrm{mg}$ HA was introduced into the mixture and stirred for another $3 \mathrm{~h}$. Finally, the hybrid nanoparticles were collected by centrifugation and repeatedly washed. The HA modified DOX@ZIF-8 was denoted DOX@ZIFHA. 


\subsection{Characterization}

The morphology of DOX@ZIF-8 before and after functionalization was observed using JEOL-2100 transmission electron microscopy (TEM) at an operating voltage of $200 \mathrm{kV}$. Fieldemission scanning electron microscopy (FESEM) was performed on a Hitachi S-4800 (Japan). An X-ray diffraction (XRD) pattern was produced on a Rigaku D/MAX-2550 PC diffractometer using monochromic $\mathrm{Cu}-\mathrm{K} \alpha$ radiation operated at $40 \mathrm{kV}$ and $80 \mathrm{~mA}$ in a $2 \theta$ range of $10-50^{\circ}$. The size distribution of DOX@ZIF-HA in different media was measured by dynamic light scattering (DLS) on a BI-200SM multiangle dynamic/static laser scattering instrument (Brookhaven, U.S.). Zeta potential measurements were performed on Malvern Zetasizer Nano ZS apparatus. A Fourier transform infrared (FTIR) spectrum was recorded using a Nicolet 6700 spectrometer.

\subsection{Drug release behavior}

Prior to the drug release experiment, the drug loading efficacy was determined. Briefly, a certain amount of DOX@ZIF-HA was incubated in diluted hydrochloric acid solution overnight and the absorption value of the supernatant solution at $480 \mathrm{~nm}$ was measured using a UV-vis spectrophotometer.

To investigate the drug release behavior, $5 \mathrm{mg}$ DOX@ZIF-HA was dispersed in $1 \mathrm{~mL}$ buffer solution with different $\mathrm{pH}$ values (pH 5.0 and 7.4), and sealed in a dialysis bag (MWCO $\sim 3500$ Da). The dialysis bag was immersed in the corresponding buffer solutions and shaken at $37^{\circ} \mathrm{C}$. The release medium was taken out for UV-vis spectrophotometer measurements and fresh medium was supplied at each time point.

\subsection{MR imaging ability}

The obtained DOX@ZIF-HA was dispersed in DI water at different $\mathrm{Fe}$ concentrations. The $T_{1}$ relaxometry of those dispersions was measured using a 0.5-T NMI20-Analyst NMR Analyzing and Imaging system (Shanghai Niumag Corporation, China). Then the $T_{1}$ relaxivity was calculated using a linear fit of the inverse $T_{1}\left(1 / T_{1}\right)$ value as a function of Fe concentration. Correspondingly, the $T_{1}$-weighted images of those dispersions were also recorded.

\subsection{Cell culture}

The human prostate carcinoma cell line (PC-3) was provided by American Type Tissue Collection (ATTC, Rockville, MD). The mouse fibroblast cell line (L929) was purchased from the Shanghai Institute of Cell Biology, Chinese Academy of Sciences (Shanghai, China). PC-3 cells and L929 cells were grown in RPMI-1640 medium and DMEM medium, respectively, supplemented with $10 \%$ FBS and $1 \%$ penicillin-streptomycin (v/v) and cultured in a humidified atmosphere with $5 \% \mathrm{CO}_{2}$ at $37{ }^{\circ} \mathrm{C}$.

\subsection{In vitro cellular uptake}

The cellular uptake of DOX-incorporated nanoparticles was first investigated using flow cytometry (FCM). PC-3 cells were detached by trypsin and seeded in a six-well plate at a density of $2 \times 10^{5}$ cells per well. After the cells were completely adhered, the medium was discarded and fresh medium containing free DOX and DOX@ZIF-HA was added. The cells were cultured for another $2 \mathrm{~h}$. Afterward, the cells were trypsinized, washed with PBS several times, and filtered with 400-mesh sieves. The cells were then suspended in PBS for FCM analysis.

The cellular uptake of DOX-incorporated nanoparticles was also inspected by confocal laser scanning microscopy (CLSM). Typically, $2 \times 10^{5}$ PC-3 cells or L929 cells were seeded in a glassbottom culture dish and incubated overnight. Then the cells were co-cultured with free DOX and DOX@ZIF-HA at a DOX concentration of $5 \mu \mathrm{g} \mathrm{mL} \mathrm{m}^{-1}$ for $2 \mathrm{~h}$. Afterwards, the medium was removed and the cells were washed with PBS several times. The cells were stained with $4 \%$ paraformaldehyde for $15 \mathrm{~min}$. Finally, the cells were directly observed using CLSM equipped with an oil lens.

For a comparative experiment, PC-3 cells or L929 cells were pre-treated with $10 \mathrm{mg} \mathrm{mL}{ }^{-1}$ HA solution for $4 \mathrm{~h}$. The other procedures were performed in the same way.

\subsection{In vitro therapeutic efficacy}

The therapeutic efficacy of DOX-incorporated carriers against PC-3 cells and L929 cells was evaluated using a standard CCK-8 assay. In brief, PC-3 cells or L929 cells were seeded into a 96-well plate at a density of $10^{4}$ cells per well. After being cultured for $24 \mathrm{~h}$, the cells were treated with free DOX, DOX@ZIF-8 and DOX@ZIF-HA at various concentrations (DOX concentrations of $0.5,1,2$ and $4 \mu \mathrm{g} \mathrm{mL}^{-1}$ ) and maintained for another $24 \mathrm{~h}$. Then the cells were washed with PBS and cultured with CCK-8 working solution for $2 \mathrm{~h}$. The absorbance at $450 \mathrm{~nm}$ of each sample was measured using a microplate reader. The cell viability was calculated by the value of the control group divided by the values of the samples. Four parallel experiments were conducted for each group.

To qualitatively observe the therapeutic efficacy, PC-3 cells were cultured and treated using the same aforementioned procedure. Then the cells were slightly rinsed with PBS and stained with Calcein-AM for $15 \mathrm{~min}$. The staining solution was discarded and the cells were imaged using inverted fluorescence microscopy.

\subsection{Statistical analysis}

All values were reported as mean \pm standard deviation. A oneway analysis of variance (ANOVA) statistical method was conducted to assess the significance of the experimental data. ${ }^{*} p<$ 0.05 and $* * p<0.01$ were considered as statistically significant.

\section{Results and discussion}

\subsection{Synthesis and characterization of DOX@ZIF-HA}

The preparation of DOX@ZIF-HA is schematically illustrated in Fig. 1. DOX was incorporated into the MOF via a one-pot reaction. Initially, DOX would interact with a $\mathrm{Zn}$ ion to form a coordination polymer, then the metal organic framework could rapidly form upon the addition of an organic linker, 2methylimidazole, on the basis of a previously reported theory. ${ }^{16}$ Subsequently, the surface of DOX@ZIF-8 was modified with 


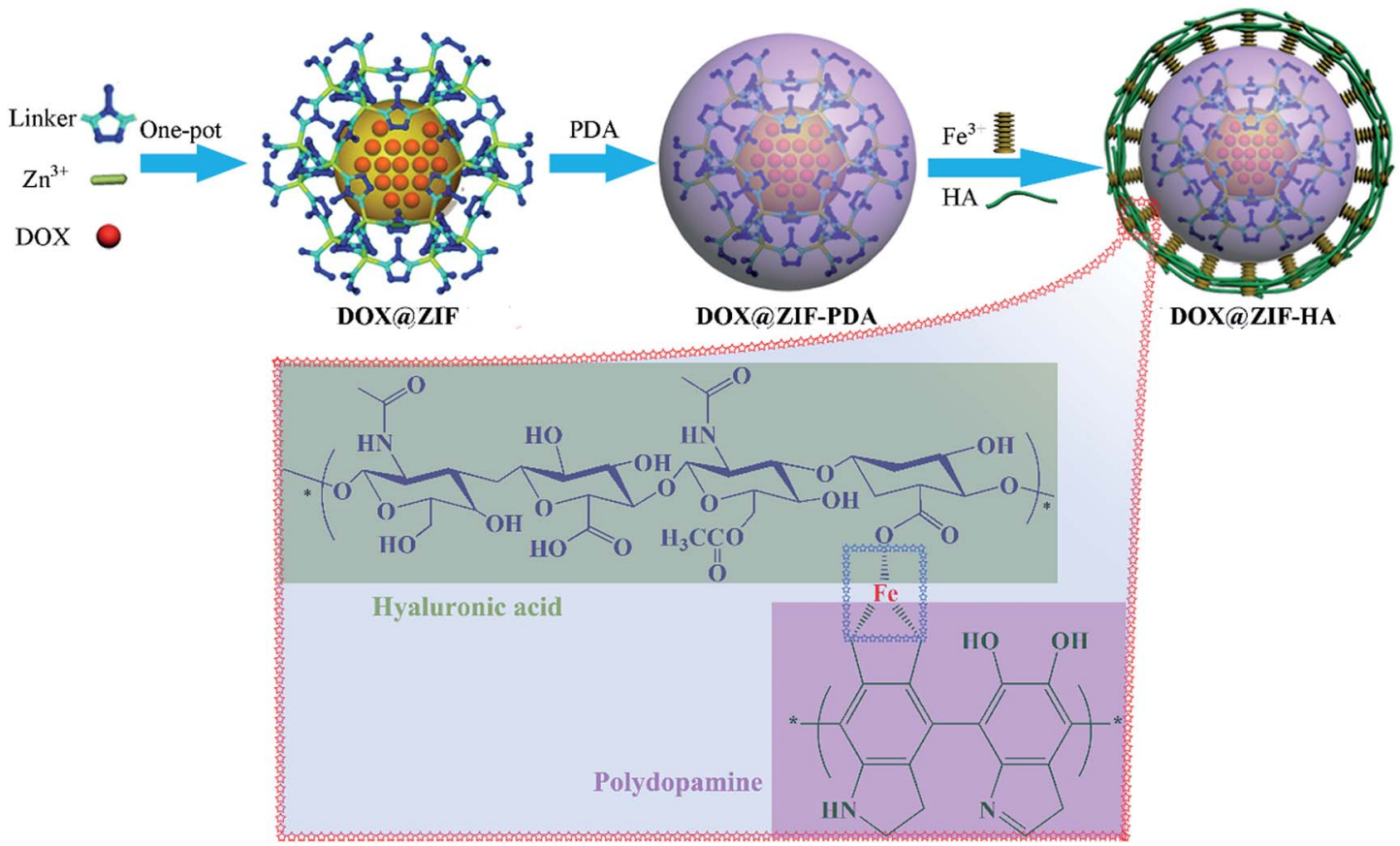

Fig. 1 A schematic diagram for the preparation of DOXCZIF-HA and the $\mathrm{Fe}^{3+}$-mediated coordination interaction between HA and PDA.

PDA, onto which an Fe ion was chelated and HA was conjugated to obtain DOX@ZIF-HA. The prepared MOF, before and after functionalization, was explored by TEM. As presented in Fig. 2B, we successfully fabricated nano-scale DOX@ZIF-8, which clearly displayed a spherical appearance with a diameter of around $150 \mathrm{~nm}$. In contrast, the bare ZIF-8 nanoparticles without DOX loading showed a typical hexagonal structure (Fig. 2A). After functionalization, the TEM images suggest that a more rough surface could be observed (Fig. 2C and D), whereas no significant change was observed in the morphology of the obtained nanoparticles when compared to pristine DOX@ZIF-8. To further confirm this, an FESEM image of DOX@ZIF-HA is presented in Fig. 2E. It can be easily seen that DOX@ZIF-HA still maintained a round shape and no apparent particle agglomeration occurred, which is beneficial for its application in drug delivery. The crystal phase was also monitored by XRD. As shown in Fig. 2F, pristine DOX@ZIF-8 exhibited well-defined diffraction peaks that correspond to the high crystallinity of
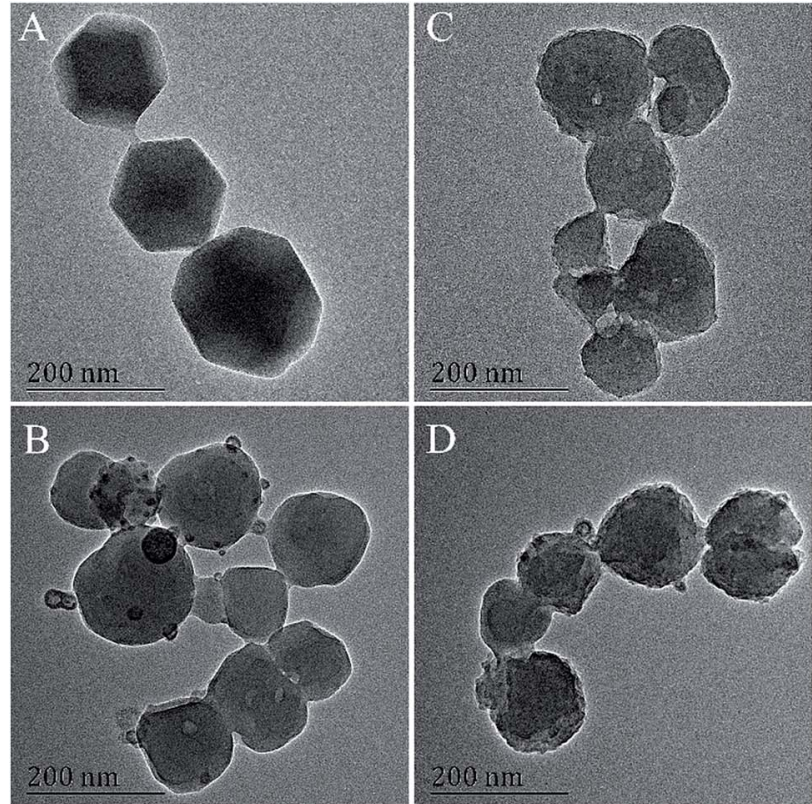
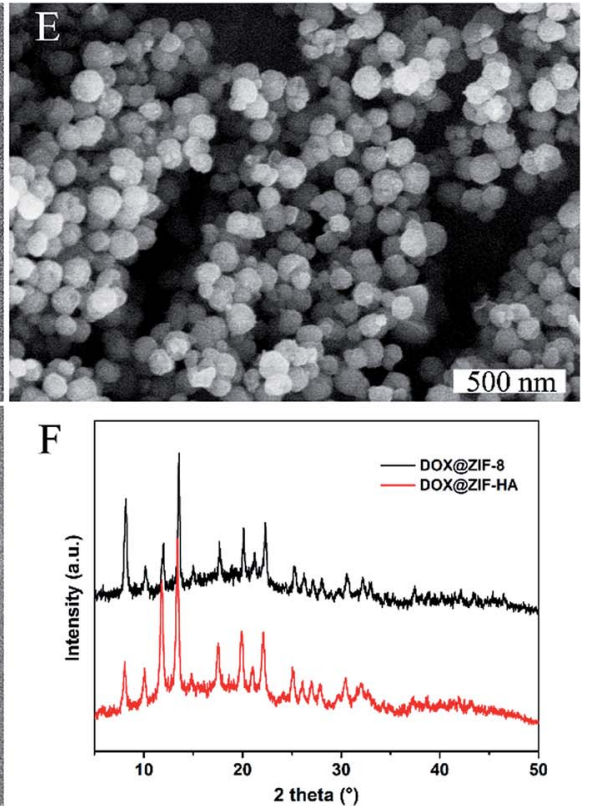

Fig. 2 TEM images of (A) pure ZIF-8, (B) DOX@ZIF-8, (C) DOX@ZIF-PDA and (D) DOX@ZIF-HA nanoparticles. (E) An FESEM image of the prepared DOX@ZIF-HA. (F) The XRD results of the prepared nanoparticles. 
ZIF-8. ${ }^{26}$ Those characteristic peaks were still retained after functionalization was completed, however, a weak broad peak emerged around $20^{\circ}$, which could be assigned to the organic shell. ${ }^{27}$

Since a good colloidal stability of nanocarriers is a prerequisite for their application, we next inspected the colloidal stability of the as-synthesized DOX@ZIF-HA in different media. DLS was applied to measure the size distribution of those dispersions (Fig. 3A). It was found that the size distribution of DOX@ZIF-HA in PBS and cell culture medium was similar to that in DI water, somewhat reflecting that no agglomeration happened even when DOX@ZIF-HA was dispersed in a solution with a high ionic strength. It was also noticed that the size of DOX@ZIF-HA was slightly increased in FBS, which might be ascribed to the nonspecific adsorbed protein on its surface. ${ }^{28}$ Meanwhile, the dispersions were stored under ambient conditions for $12 \mathrm{~h}$, and the obtained photograph indicated that no large particles appeared in the dispersions (Fig. 3B), further confirming the colloidal stability of DOX@ZIF-HA in different media.

Zeta potential measurements were performed to detect the surface charge of the prepared nanoparticles. Fig. 4A demonstrates that the as-synthesized DOX@ZIF-8 displayed a positively-charged surface with a zeta potential of $27.1 \mathrm{mV}$, while PDA modification gives the hybrid nanoparticles a negativelycharged surface owing to the abundant phenolic groups of PDA. ${ }^{29}$ After the attachment of acid mucopolysaccharide HA, ${ }^{30}$ the obtained DOX@ZIF-HA still maintained a highly negative potential with a value of $-30.2 \mathrm{mV}$. Logically, the higher negative potential could ensure good colloidal stability of the nanomaterials under physiological conditions, ${ }^{31}$ resulting in good colloidal stability of DOX@ZIF-HA as illustrated above. Afterwards, the chemical composition of the prepared nanoparticles was also investigated using FTIR. As depicted in Fig. $4 \mathrm{~B}$, the spectrum of pure ZIF-8 appeared to be typical, and the 1574 and $1145 \mathrm{~cm}^{-1}$ bands belong to $\mathrm{C}=\mathrm{N}$ stretching vibrations. Moreover, the strong peak at $1077 \mathrm{~cm}^{-1}$, assigned to the $\mathrm{C}-\mathrm{N}$ stretching of the imidazole units, was observed in both pure ZIF-8 and the other nanoformulations. ${ }^{32}$ Obviously, the bands in the range of $2800-3200 \mathrm{~cm}^{-1}$ resulted from $\mathrm{C}-\mathrm{H}$ vibrations in the backbone ring structure. ${ }^{33} \mathrm{~A}$ new peak at
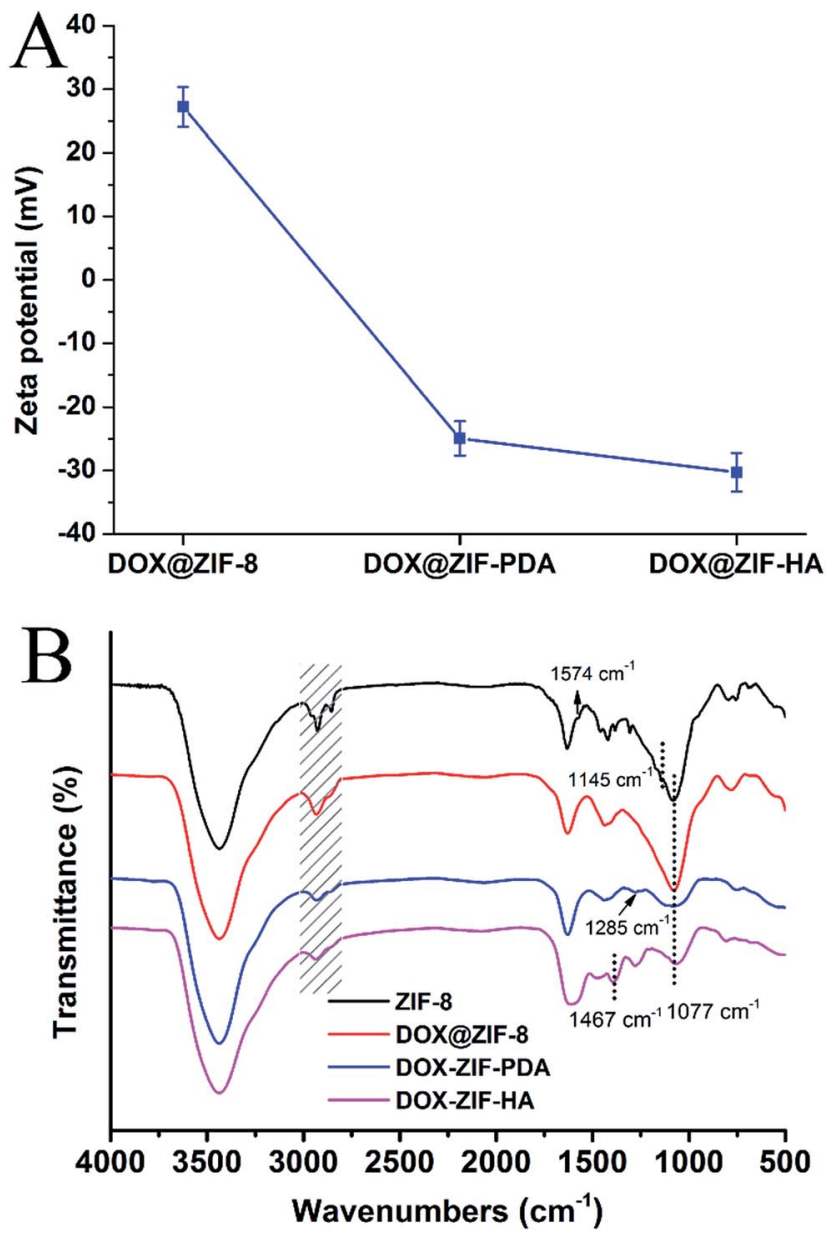

Fig. 4 (A) Surface zeta potential and (B) FTIR spectra of the prepared nanoparticles in different stages.

$1285 \mathrm{~cm}^{-1}$, corresponding to the $\mathrm{C}-\mathrm{O}$ vibration from the phenolic hydroxyl group, was clearly observed after the modification with $\mathrm{PDA}^{34}$ and the sharp peak at $1077 \mathrm{~cm}^{-1}$ was weakened and broadened at the same time. Besides, the typical absorption peaks of the amide bond were also detected in the spectrum of DOX@ZIF-HA because of the $N$-acetylglucosamine of HA. Specifically, three adjacent peaks at 1610, 1476 and

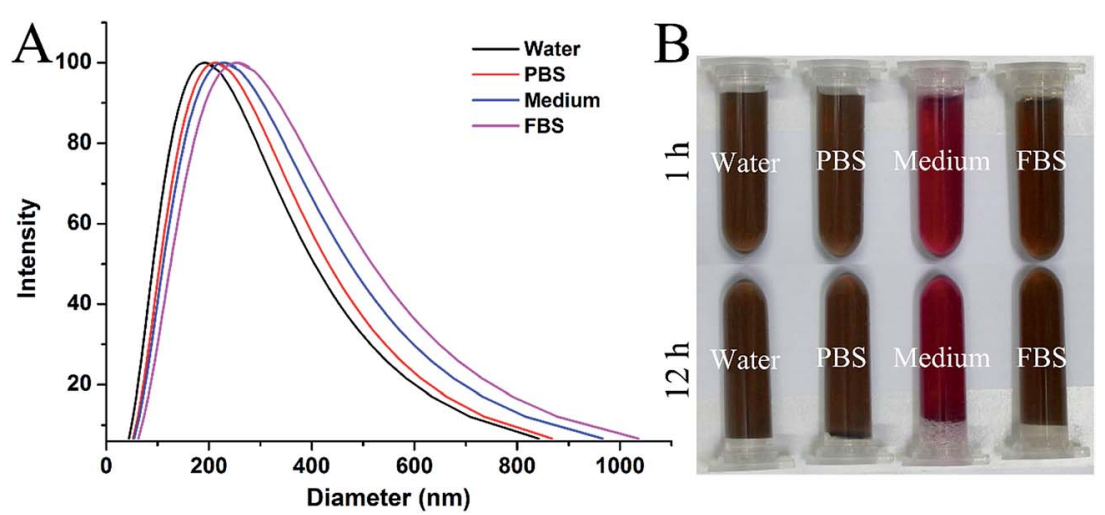

Fig. 3 (A) The size distribution of DOX@ZIF-HA dispersed in different media, as measured by DLS. (B) Photographs of different DOX@ZIF-HA dispersions stored under ambient conditions for $1 \mathrm{~h}$ and $12 \mathrm{~h}$. 
$1394 \mathrm{~cm}^{-1}$ were attributed to the absorption of the amide I, II and III bands, ${ }^{35}$ respectively. When combined with the above discussion, it can be concluded that the designed hybrid nanoplatform was successfully fabricated.

\subsection{Drug release property}

After the successful construction of DOX@ZIF-HA was verified, the release behavior of the incorporated DOX was successively investigated. The loading efficacy was determined to be $8.92 \pm$ $0.53 \%$. Fig. 5 shows the release kinetics of DOX from DOX@ZIFHA under different $\mathrm{pH}$ conditions. The data suggest that the release of DOX from the nanocarrier demonstrated a sustained nature regardless of the $\mathrm{pH}$ condition. In particular, a $\mathrm{pH}$ dependent drug release behavior was observed. It can be seen that $34 \%$ of DOX was released from DOX@ZIF-HA in the first $6 \mathrm{~h}$ under acidic conditions ( $\mathrm{pH}$ 5.0), while the released amount of DOX was only $7.7 \%$ under physiological $\mathrm{pH}$ conditions over the same time period. At the end, the cumulative amount of DOX released at $\mathrm{pH} 5.0$ and 7.4 was $70.1 \%$ and $9.8 \%$, respectively. The $\mathrm{pH}$-dependent release behavior is reasonable owing to the dissolution mechanism of the nanoscale ZIF-8 structure. It has been widely demonstrated that the coordination interaction between $\mathrm{Zn}^{2+}$ and imidazole would be disrupted under lower $\mathrm{pH}$ conditions, ${ }^{36}$ which then encourages the locked DOX to diffuse out. Another potential reason lies in the better hydrophilicity of DOX under acidic conditions, when compared to that under neutral conditions. ${ }^{37}$ Anyway, there is no doubt that the rapid release of $\mathrm{DOX}$ at $\mathrm{pH} 5.0$ could dramatically improve the delivery efficacy considering the more acidic tumor microenvironment when compared to that of normal tissues.

\subsection{Targeted cellular uptake}

Apart from the stimuli-responsive drug release properties, the targeted drug delivery could further enhance the chemotherapeutic efficacy. ${ }^{38}$ The targeting ability of DOX@ZIF-HA toward the prostate cancer cell line PC-3 was first evaluated by flow cytometry. The PC-3 cells were treated with different

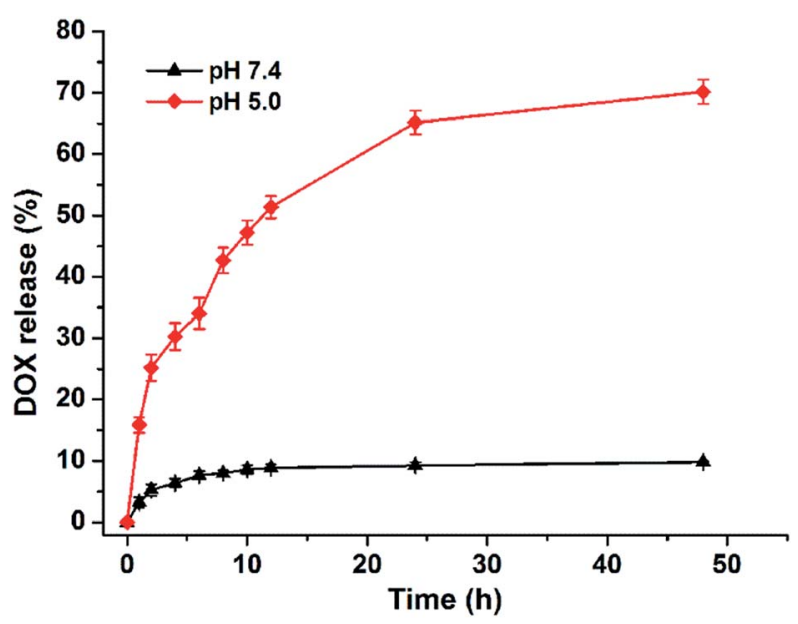

Fig. 5 The cumulative release curve of DOXaZIF-HA under different $\mathrm{pH}$ conditions. concentrations of DOX@ZIF-HA for $2 \mathrm{~h}$ and then subjected to the FCM test. The cells incubated with PBS were used as a control and the cells pre-treated with free HA were used for comparison. The data demonstrated that the intracellular fluorescence intensity was distinctly improved with the increase of DOX@ZIF-HA concentration (Fig. 6A). It is worth noting that the mean fluorescence of cells pre-treated with free HA was higher than that of cells directly treated with DOX@ZIF-HA at the same concentration. Specifically, the value decreased from 217.37 to 193.19 at a concentration of $100 \mu \mathrm{g} \mathrm{mL}^{-1}$ after pretreatment with free HA (Fig. 6B). Considering the widely reported overexpressed CD44 receptor on PC-3 cells, ${ }^{39,40}$ the value decrease was because the free HA would inhibit the overexpressed CD44 receptor on PC-3 cells. Thus, it can be deduced that the prepared DOX@ZIF-HA could efficiently target PC-3 cells.

Moreover, the cells were incubated with free DOX and DOX@ZIF-HA for $2 \mathrm{~h}$ and CLSM was applied to visually observe their intracellular uptake. As presented in Fig. 7A, the red fluorescence of DOX completely overlapped with the blue fluorescence of the DAPI-stained cell nucleus, while the red spot of DOX@ZIF-HA was mainly distributed in the cell cytoplasm and localized around the cell nucleus. As a small molecule drug,
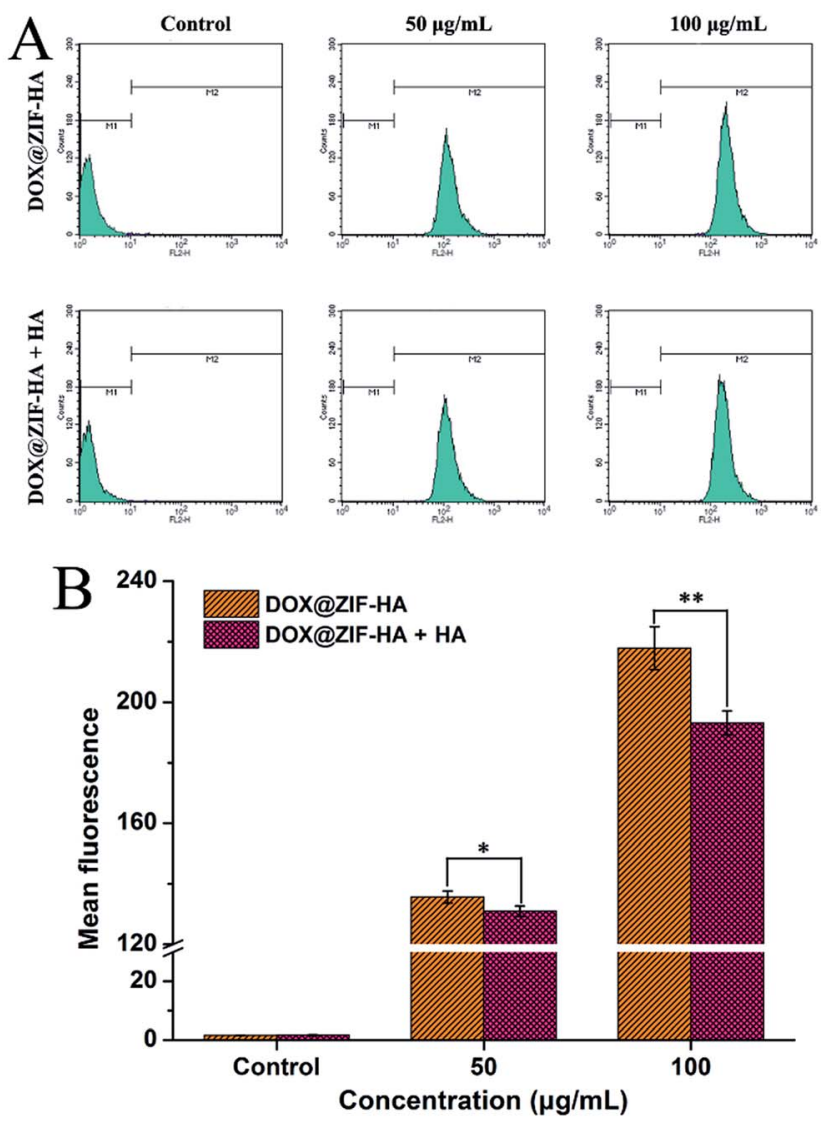

Fig. 6 (A) Flow cytometric analysis of HeLa cells treated with different concentrations of DOX@ZIF-HA for $2 \mathrm{~h}$, free HA was used to inhibit CD44 receptors on $\mathrm{PC}-3$ cells for comparison. (B) Mean fluorescence density in PC -3 cells after the corresponding treatment (* for $p<0.05$ and $* *$ for $p<0.01$ ). 

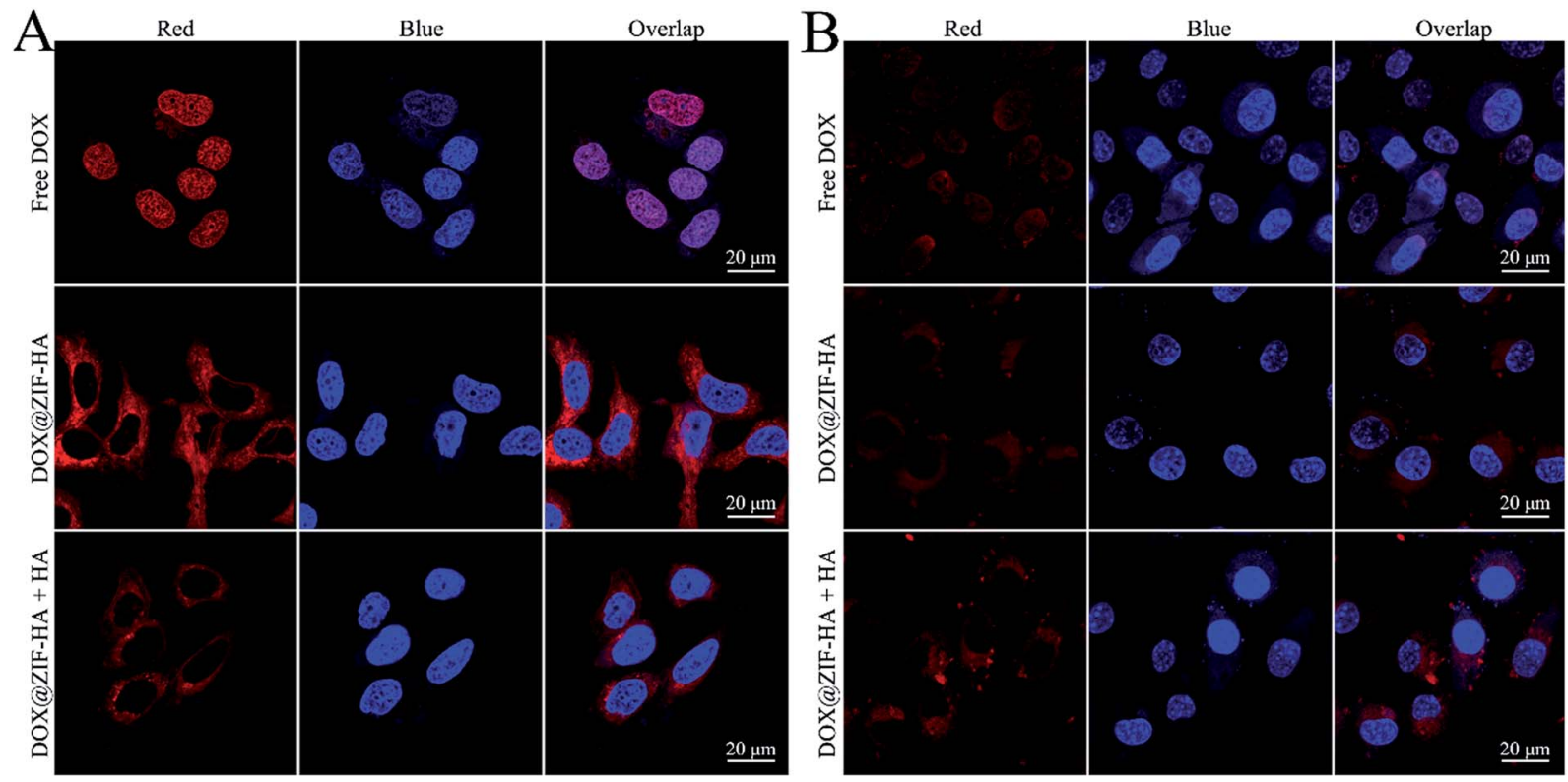

Fig. 7 CLSM images of (A) PC-3 cells and (B) L929 cells, treated with free DOX and DOX@ZIF-HA for $2 \mathrm{~h}$, the cells pre-treated with HA were observed for comparison. The blue and red fluorescence represents the DAPI-stained cell nucleus and DOX, respectively.

DOX entered into PC-3 cells via passive diffusion, thus resulting in rapid entrance into the cell nucleus. In contrast, the nanocarrier was actively transported into the cell interior by enclosing it in vesicles. ${ }^{41}$ So the retention of DOX@ZIF-HA in the cytoplasm could facilitate the sustained release of DOX and maintain a sufficient drug concentration in cancer cells. ${ }^{42}$ In addition, the enhanced uptake of DOX@ZIF-HA by the PC-3 cells was also confirmed. It can be seen that the fluorescence in the case of DOX@ZIF-HA was much stronger than that of the cells pre-treated with free HA. This result should be attributed to the inhibition of the overexpressed CD44 receptors on the PC3 cell surface by free HA, leading to the subdued targeting ability of DOX@ZIF-HA.

Normal cells L929 were also employed to further confirm the targeting ability of DOX@ZIF-HA toward specific cancer cells. As seen in Fig. 7B, the free DOX also directly diffused into the cell nucleus. Meanwhile, it also can be observed that red spots of DOX@ZIF-HA accumulated in the cytoplasm. Notably, the fluorescence intensity of L929 cells was clearly lower than that of PC-3 cells. This tendency could probably be attributed to the negative surface of DOX@ZIF-HA. Meanwhile, it is worth noting that the treatment of free HA didn't reduce the internalization of DOX@ZIF-HA, showing the different uptake behavior with CD44-overexpressed PC-3 cells. Predictably, the targeting ability of DOX@ZIF-HA would lose its "magic" toward a normal cell line due to the lower expression of the CD44 receptor on L929. Taken together, the above results forcefully affirm that DOX@ZIF-HA could efficiently enhance intracellular uptake and deliver more of the drug into PC-3 cells.

\subsection{In vitro killing efficacy of $\mathrm{PC}-3$ cells}

After confirming the targeting ability of DOX@ZIF-HA, its therapeutic activity against PC-3 cells was assessed using the
CCK-8 assay. The cells were treated with different formulations for $24 \mathrm{~h}$ and un-treated cells were used as a control. As shown in Fig. 8A, the viability of treated PC-3 cells clearly decreased with increased DOX concentration. Specifically, the toxicity of DOX@ZIF-HA was significantly higher than that of free DOX at the same concentration. For instance, at a DOX concentration of $1 \mu \mathrm{g} \mathrm{mL}{ }^{-1}$, the cell viability for free DOX and DOX@ZIF-HA was $65.61 \%$ and $48.59 \%$, respectively. This is mainly due to the fact that the efficient internalization of the targeted carrier DOX@ZIF-HA could improve the intracellular DOX concentration. ${ }^{43}$ Furthermore, the inhibition assay was also performed to demonstrate the targeting ability. It was observed that the toxicity of DOX@ZIF-HA clearly decreased in the presence of free HA. In particular, the cell viability of DOX@ZIF-HA was $20.22 \%$ at a DOX concentration of $4 \mu \mathrm{g} \mathrm{mL}^{-1}$, while the value increased to $26.56 \%$ after pre-treatment with free HA. Undoubtedly, the decrease in therapeutic efficacy was caused by the inhibition of free HA toward the targeting ability of DOX@ZIF-HA. To further demonstrate the discrepancy, live cell staining was performed. Fig. 8C shows the fluorescent imaging of PC-3 cells treated with different formulations. It can be seen that the strong green fluorescence was overspread on the culture well for the control group, while the amount of live cells sharply decreased after incubation with different treatments. In particular, DOX@ZIF-HA treated cells exhibited a higher mortality rate as compared to free DOX-treated PC-3 cells and the inhibited group, suggesting the same tendency as the quantitative results.

For comparison, L929 cells were also subjected to the same treatments and the data are presented in Fig. 8B. Although the cell viabilities decreased with elevated DOX concentrations, it should be noted that the survival rate of L929 cells was distinctly lower than that of PC-3 cells at an equivalent DOX 

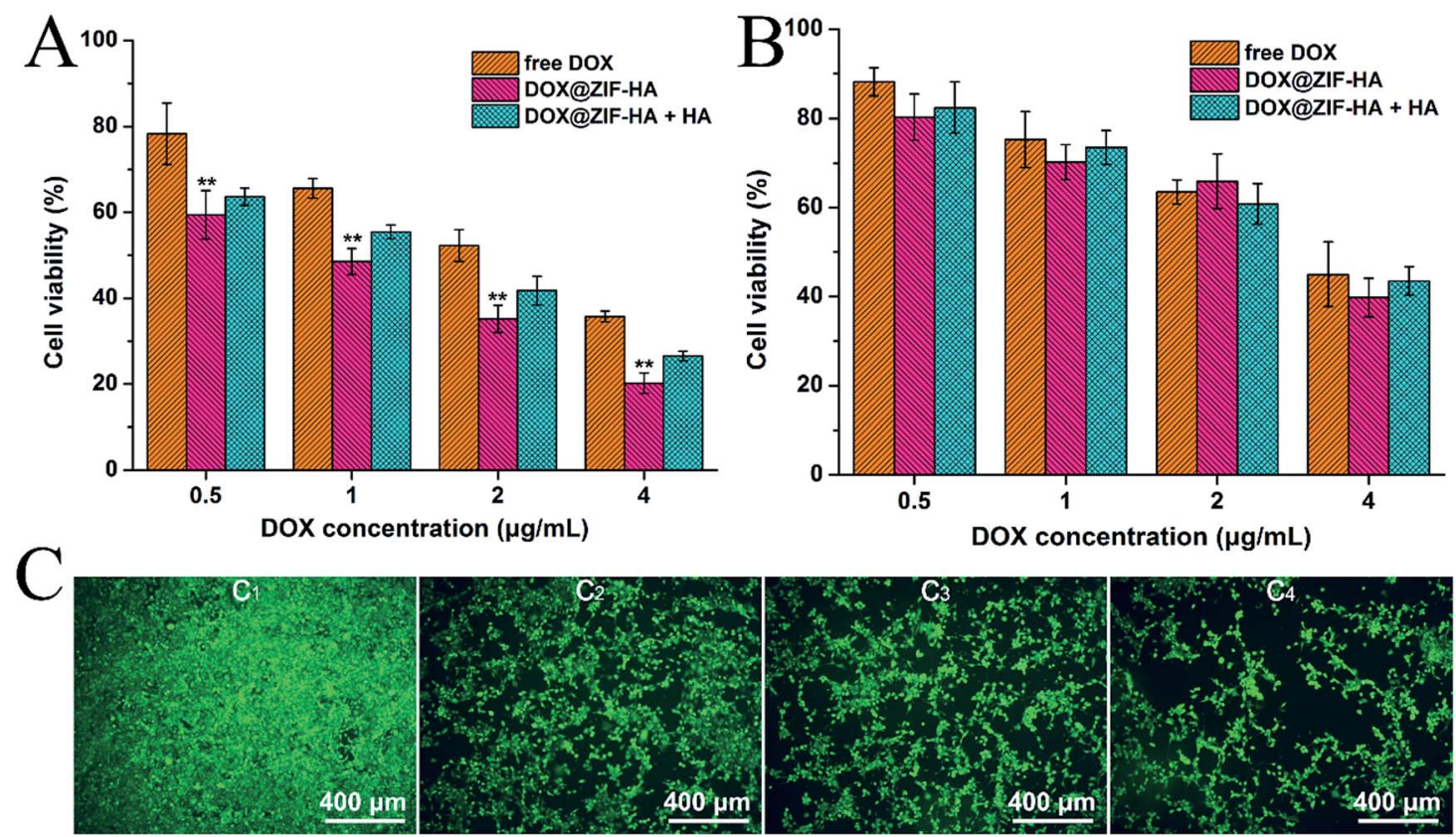

Fig. 8 The CCK-8 assay of (A) PC-3 cells and (B) L929 cells, treated with free DOX and DOX@ZIF-HA at different DOX concentrations for $24 \mathrm{~h}$. (C) Live cell staining (Calcein-AM) of PC-3 cells treated with $\left(C_{1}\right)$ culture medium, $\left(C_{2}\right)$ free DOX, $\left(C_{3}\right)$ DOX $\left(a Z I F-H A\right.$ plus free HA and $\left(C_{4}\right)$ DOX@ZIF$\mathrm{HA}$, at a DOX concentration of $4 \mu \mathrm{g} \mathrm{mL} \mathrm{m}^{-1}$.

concentration. Not surprisingly, the inhibition of free HA exerted negligible influence on the therapeutic efficacy of DOX@ZIF-HA. Specifically, at a DOX concentration of $4 \mu \mathrm{g} \mathrm{mL}{ }^{-1}$, the viability for free DOX, DOX@ZIF-HA and DOX@ZIF-HA plus free HA treated L929 cells was $45.04 \%$, $39.84 \%$ and $43.56 \%$, respectively. It could be expected that the lower toxicity of DOX@ZIF-HA against L929 would alleviate its side effects toward normal organs, somewhat highlighting the intrinsic merit of actively targeted nanocarriers. Therefore, the above results suggest that the prepared DOX@ZIF-HA could be efficiently applied as an actively targeted nanocarrier to improve chemotherapeutic efficacy.

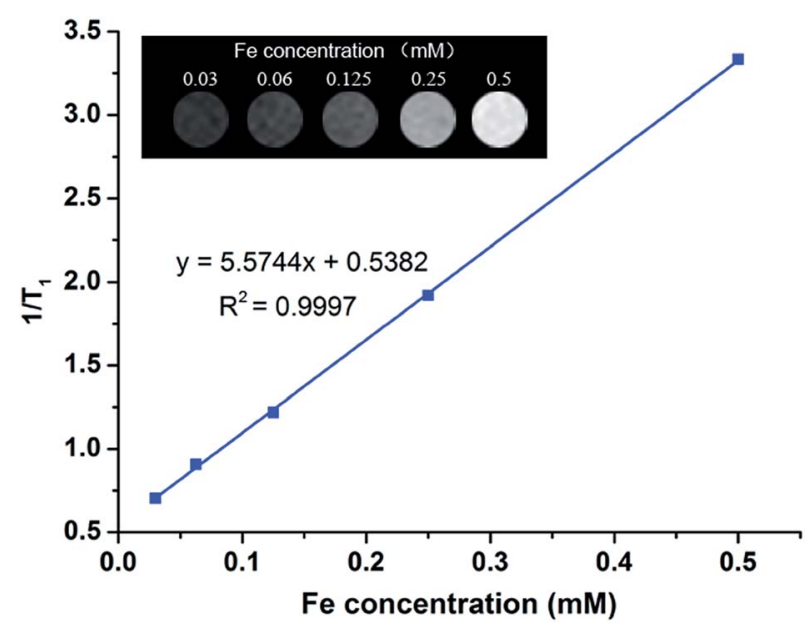

Fig. 9 The in vitro MR imaging ability of DOXaZIF-HA. The linear fitting of $1 / T_{1}$ as a function of Fe concentration. The inset pictures represent the corresponding $T_{1}$-weighted MR images of DOX@ZIF-HA at different Fe concentrations.

\subsection{In vitro $\mathrm{MR}$ imaging ability}

Apart from mediating the conjugation of HA, the chelation of $\mathrm{Fe}^{3+}$ by PDA could also be used as a $T_{1}$ contrast agent. ${ }^{44,45}$ So the MR imaging ability of DOX@ZIF-HA was evaluated in vitro. As expected, the longitudinal relaxation time $\left(T_{1}\right)$ decreased correlatively with increased Fe concentration (Fig. 9). The $r_{1}$ value was calculated to be $5.57 \mathrm{mM}^{-1} \mathrm{~s}^{-1}$, which is higher than a clinically used MRI contrast agent Gd-DTPA. Correspondingly, the MR images of DOX@ZIF-HA dispersions became brighter with the increase of Fe concentration (inset photos in Fig. 9), implying their favorable MR imaging ability. Thus, the DOX@ZIF-HA could also act as an excellent contrast agent for MR imaging.

\section{Conclusion}

In summary, DOX-doped MOF nanoparticles were prepared via a one-pot reaction and successively anchored with $\mathrm{Fe}^{3+}$ and $\mathrm{HA}$ for simultaneous targeted drug delivery and MR imaging. The successful construction of DOX@ZIF-HA was confirmed by a series of physicochemical characterization techniques. The incorporated DOX could be released from the nanocarrier in a sustained and $\mathrm{pH}$-sensitive manner. The inhibition experiment also demonstrated that the targeting ability of DOX@ZIF-8-HA toward CD44 overexpressed PC-3 cells could efficiently improve its intracellular uptake and further enhance the in vitro chemotherapeutic efficacy as compared to free DOX. Moreover, the chelation of $\mathrm{Fe}^{3+}$ endowed DOX@ZIF-HA with a favorable contrast ability for MR imaging. Overall, the developed DOX@ZIF-HA could be applied as a potential theranostic agent for chemotherapy of CD44 overexpressed PC-3 cells and MR imaging. 


\section{Conflicts of interest}

There are no conflicts to declare.

\section{Acknowledgements}

This study was supported by three Science and Technology planning Projects of Guangdong Province (No. 2013B051000050, No. 2014A020212538 and No. 2016A020215175), the Natural Science Foundation of Guangdong Province (No. 2016A030313583), the Medical Scientific Research Foundation of Guangdong Province (No. A2016555), the Science and Technology planning Project of Guangzhou (No. 201704020070), and the Outstanding Youths Development Scheme of Nanfang Hospital, Southern Medical University (No. 2015J005).

\section{References}

1 P. Couvreur, Adv. Drug Delivery Rev., 2013, 65, 21-23.

2 C. Yao, P. Wang, X. Li, X. Hu, J. Hou, L. Wang and F. Zhang, Adv. Mater., 2016, 28, 9341-9348.

3 V. G. Deepagan, S. Kwon, D. G. You, V. Q. Nguyen, W. Um, H. Ko, H. Lee, D. G. Jo, Y. M. Kang and J. H. Park, Biomaterials, 2016, 103, 56-66.

4 K. Yang, L. Feng and Z. Liu, Adv. Drug Delivery Rev., 2016, 105, 228-241.

5 J. Wen, K. Yang, F. Liu, H. Li, Y. Xu and S. Sun, Chem. Soc. Rev., 2017, 46, 6024-6045.

6 W. Cui, J. Li and G. Decher, Adv. Mater., 2016, 28, 1302-1311. 7 Y. Chen and J. Shi, Adv. Mater., 2016, 28, 3235-3272.

8 E. B. Ehlerding, F. Chen and W. Cai, Adv. Sci., 2016, 3, 1600122.

9 P. Huang, Y. Chen, H. Lin, L. Yu, L. Zhang, L. Wang, Y. Zhu and J. Shi, Biomaterials, 2017, 125, 23-37.

10 D. Maspoch, D. Ruiz-Molina and J. Veciana, Chem. Soc. Rev., 2007, 36, 770-818.

11 J. Della Rocca, D. Liu and W. Lin, Acc. Chem. Res., 2011, 44, 957-968.

12 W. Cai, C. C. Chu, G. Liu and Y. X. Wang, Small, 2015, 11, 4806-4822.

13 C. Y. Sun, C. Qin, X. L. Wang, G. S. Yang, K. Z. Shao, Y. Q. Lan, Z. M. Su, P. Huang, C. G. Wang and E. B. Wang, Dalton Trans., 2012, 41, 6906-6909.

14 L. He, T. T. Wang, J. P. An, X. M. Li, L. Y. Zhang, L. Li, G. Z. Li, X. T. Wu, Z. M. Su and C. G. Wang, CrystEngComm, 2014, 16, 3259-3263.

15 Z. F. Wang, X. J. Tang, X. X. Wang, D. D. Yang, C. Yang, Y. B. Lou, J. X. Chen and N. Y. He, Chem. Commun., 2016, 52, 12210-12213.

16 H. Q. Zheng, Y. N. Zhang, L. F. Liu, W. Wan, P. Guo, A. M. Nystrom and X. D. Zou, J. Am. Chem. Soc., 2016, 138, 962-968.

17 M. Zheng, S. Liu, X. G. Guan and Z. G. Xie, ACS Appl. Mater. Interfaces, 2015, 7, 22181-22187.

18 Q. Chen and Z. Liu, Adv. Mater., 2016, 28, 10557-10566.

19 A. R. Chowdhuri, D. Laha, S. Pal, P. Karmakar and S. K. Sahu, Dalton Trans., 2016, 45, 18120-18132.
20 R. X. Bian, T. T. Wang, L. Y. Zhang, L. Li and C. G. Wang, Biomater. Sci., 2015, 3, 1270-1278.

21 F. Ravar, E. Saadat, M. Gholami, P. Dehghankelishadi, M. Mahdavi, S. Azami and F. A. Dorkoosh, J. Controlled Release, 2016, 229, 10-22.

22 Q. Zhao, S. Wang, Y. Yang, X. Li, D. Di, C. Zhang, T. Jiang and S. Wang, Mater. Sci. Eng., C, 2017, 78, 475-484.

23 D. D. Gurav, A. S. Kulkarni, A. Khan and V. S. Shinde, Colloids Surf., B, 2016, 143, 352-358.

24 H. Lee, S. M. Dellatore, W. M. Miller and P. B. Messersmith, Science, 2007, 318, 426-430.

25 J. Han, W. Park, S. J. Park and K. Na, ACS Appl. Mater. Interfaces, 2016, 8, 7739-7747.

26 H. P. Jing, C. C. Wang, Y. W. Zhang, P. Wang and R. Li, RSC Adv., 2014, 4, 54454-54462.

27 B. Q. Mao, Q. D. An, B. Zhai, Z. Y. Xiao and S. R. Zhai, $R S C$ Adv., 2016, 6, 47761-47770.

28 Y. S. Lin, N. Abadeer and C. L. Haynes, Chem. Commun., 2011, 47, 532-534.

29 F. F. Cheng, J. J. Zhang, F. Xu, L. H. Hu, E. S. Abdel-Halim and J. J. Zhu, J. Biomed. Nanotechnol., 2013, 9, 1155-1163.

30 Z. H. Zhu, D. Y. Li, Y. N. Li, X. G. Yang and W. S. Pan, RSC Adv., 2017, 7, 23942-23953.

31 X. H. Liu, C. H. Gao, J. H. Gu, Y. F. Jiang, X. L. Yang, S. Y. Li, W. Gao, T. An, H. Q. Duan, J. W. Fu, Y. S. Wang and X. Y. Yang, ACS Appl. Mater. Interfaces, 2016, 8, 27622-27631.

32 Z. Tian, X. Yao, K. Ma, X. Niu, J. Grothe, Q. Xu, L. Liu, S. Kaskel and Y. Zhu, ACS Omega, 2017, 2, 1249-1258.

33 L. F. Ren, X. J. Liu, Q. Wang, L. J. Zhang, G. Y. Deng, F. Zhou and J. Lu, Dalton Trans., 2017, 46, 2204-2213.

34 W. X. Mao, X. J. Lin, W. Zhang, Z. X. Chi, R. W. Lyu, A. M. Cao and L. J. Wan, Chem. Commun., 2016, 52, 7122-7125.

35 Y. M. Hu, J. D. Chen, T. T. Fan, Y. J. Zhang, Y. Zhao, X. T. Shi and Q. Q. Zhang, Colloids Surf., B, 2017, 157, 93-100.

36 C. C. Zheng, Y. Wang, S. Z. F. Phua, W. Q. Lim and Y. L. Zhao, ACS Biomater. Sci. Eng., 2017, 3, 2223-2229.

37 S. G. Wang, Y. L. Wu, R. Guo, Y. P. Huang, S. H. Wen, M. W. Shen, J. H. Wang and X. Y. Shi, Langmuir, 2013, 29, 5030-5036.

38 L. L. Dai, J. J. Liu, Z. Luo, M. H. Li and K. Y. Cai, J. Mater. Chem. B, 2016, 4, 6758-6772.

39 K. A. Iczkowski, Am. J. Transl. Res., 2011, 3, 1-7.

40 P. Kesharwani, S. Banerjee, S. Padhye, F. H. Sarkar and A. K. Iyer, Biomacromolecules, 2015, 16, 3042-3053.

41 F. Zhao, Y. Zhao, Y. Liu, X. L. Chang, C. Y. Chen and Y. L. Zhao, Small, 2011, 7, 1322-1337.

42 W. Feng, W. Nie, C. L. He, X. J. Zhou, L. Chen, K. X. Qiu, W. Z. Wang and Z. Q. Yin, ACS Appl. Mater. Interfaces, 2014, 6, 8447-8460.

43 M. H. Yu, S. Jambhrunkar, P. Thorn, J. Z. Chen, W. Y. Gu and C. Z. Yu, Nanoscale, 2013, 5, 178-183.

44 K. Y. Ju, J. W. Lee, G. H. Im, S. Lee, J. Pyo, S. B. Park, J. H. Lee and J. K. Lee, Biomacromolecules, 2013, 14, 3491-3497.

45 K. Y. Ju, S. Lee, J. Pyo, J. Choo and J. K. Lee, Small, 2015, 11, 84-89. 